\title{
Kennzahlen für den Feinheitsgrad des Lösses
}

\author{
Von ERnst Schönhals, Wiesbaden
}

Mit 10 Abb.

$\mathrm{Z}$ us a mmenf assung. Bei der Untersuchung des Lösses spielt die Korngrößenzusammensetzung eine wesentliche Rolle, da man aus der Änderung der Körnung bzw. aus der Feinheit wichtige Schlüsse auf die Entstehung des äolischen Sedimentes ziehen kann. Bei der Ermittlung von Unterschieden in der Feinheit mußte man sich bisher darauf beschränken, einzelne Fraktionen untereinander zu vergleichen. Dieses Verfahren konnte natürlich zu keinen exakten Ergebnissen führen. Die beschriebene Methode erlaubt nun, den Feinheitsgrad in einer einzigen Zahl, der sog. Kennzahl anzugeben. Mit Hilfe der aus den Gewichtsprozenten schnell zu errechnenden Kennzahlen, die allerdings keine absoluten, sondern relative Werte darstellen, ist es möglich, die Änderungen des Feinheitsgrades quantitativ zu erfassen und in Abhängigkeit von anderen Faktoren graphisch darzustellen.

An mehreren Beispielen wird die Anwendung der Kennzahlen näher erläutert. Bei zwei Lößprofilen konnte durch die Kennzahlen eine Abnahme der Feinheit von unten nach oben nachgewiesen werden. Ob dies auch bei anderen Lößprofilen der Fall ist, müssen weitere Untersuchungen zeigen. An drei anderen Beispielen wurde die Änderung der Feinheit mit der Entfernung vom Auswehungsgebiet untersucht. In den drei Gebieten nimmt die Feinheit mit der Entfernung zu und zwar steigt der Feinheitsgrad linear mit dem Logarithmus der Entfernung. Die schnellste Zunahme des Feinheitsgrades erfolgt in der Randzone des Auswehungsgebietes, wo infolgedessen auch die größten Lößmächtigkeiten angetroffen werden. Die Zunahme der Feinheit vom Auswehungs- bis in das Ablagerungsgebiet kann durch eine einzige Kurve zum Ausdruck gebracht werden.

$\mathrm{S} \mathrm{u} \mathrm{m} \mathrm{m} \mathrm{a} \mathrm{r} \mathrm{y.} \mathrm{On} \mathrm{studying} \mathrm{the} \mathrm{loess,} \mathrm{the} \mathrm{particle-size} \mathrm{distribution} \mathrm{is} \mathrm{of} \mathrm{great} \mathrm{importance,} \mathrm{as}$ from the variations in its fineness essential inferences can be drawn concerning the eolian sediments. To find out the differences in the fineness one was restricted so far to comparing the single fractions. Of course, this method could not give exact results. The described new method now allows to indicate the degree of fineness by a single factor, the so-called "Kennzahl" ("characteristic figure"). By means of the "Kennzahl" - quickly calculated from the weightpercentage - , which, however, do not give absolute but relative values, it is possible to find out the variation of the degree of fineness in quantity and to plot it graphically against other factors.

The use of the „Kennzahl“ is illustrated by several examples. In two loess profiles it was possible, by means of the "Kennzahl" to prove a decrease of the fineness from bottom to top. Further studies will show, whether this is also the case in other loess profiles. By means of three other examples the variation of fineness was examined as compared with the distance from the original area, from where the loess was blown along. In those three districts the finer fractions increase with the distance, and there the degree of fineness increases in a linear manner with the logarithm of the distance. The most rapid increase of the degree of fineness is in the neighbourhood of the origin area where consequently the greatest thickness of loess is to be found. The increase of the fineness from the origin area to the deposits can be expressed by a single curve.

\section{E in le it ung}

Wohl bei keinem anderen Lockergestein spielt die Korngrößenzusammensetzung eine so große Rolle wie beim Löß. Seine durch die äolische Entstehung bedingte charakteristische Körnung wird daher fast bei jeder Untersuchung ermittelt, so z. B. wenn es darum geht, festzustellen, aus welcher Richtung der Staub angeweht wurde, oder beim Vergleich der in einem Lößprofil entnommenen Proben. Auch eine Beimischung von Löß in anderen Bodenarten ist in den meisten Fällen durch eine Korngrößenbestimmung zu erkennen. Gegenüber der Körnungsanalyse treten daher chemische und mineralogische Untersuchungsmethoden zurück.

Die Ergebnisse der mechanischen Analyse werden bekanntlich in Gewichtsprozenten angegeben und zur besseren Übersicht häufig als Kurven dargestellt. Die einfachste Art 
der graphischen Darstellung ist die Kornverteilungskurve, auch Häufigkeits- und Differentialkurve genannt. Hierbei werden im allgemeinen auf der Abszisse die unterschiedenen Korngrößengruppen mit gleichen Abständen und auf der Ordinate die dazugehörigen Gewichtsprozente aufgetragen. Durch Verbinden der einzelnen Werte erhält man die Kornverteilungskurve, die in der Fraktion $0,01-0,05 \mathrm{~mm}$ ein ausgeprägtes Maximum aufweist ${ }^{1}$ ) (vgl. die zahlreichen Abbildungen bei J. BFHR \&\& R. KöHLER 1930 und A. Scheidig 1934).

Es sei jedoch in diesem Zusammenhang erwähnt, daß diese Darstellung nicht ganz richtig ist, und zwar aus folgenden Gründen: Die Korngruppen werden - wie bereits erwähnt - auf der Abszisse mit g leichen Abständen aufgetragen, obgleich sie nicht gleich groß sind. Die Fläche der über den einzelnen Kornspielräumen gezeichneten Rechtecke entspricht dann nicht den Gewichtsprozenten der betreffenden Fraktionen, eine Voraussetzung, die aber erfüllt sein muß, wenn die durch Ausgleich der Rechtecke gewonnene Kurve die tatsächliche Kornverteilung wiedergeben soll. Es wäre daher bei der früher allgemein benutzten und zuweilen auch heute noch anzutreffenden Darstellungsweise eine Berechnung der jeweiligen Ordinate erforderlich, damit die Rechtecke über den in gleicher Länge aufgetragenen Abszissenabständen in ihrer Fläche mit den Gewichtsprozenten übereinstimmen.

Die zweite, heute allgemein übliche Form der Darstellung ist die Summenkurve (Integralkurve). Hierbei wird die Abszisse nach den gewählten Fraktionen unterteilt und auf der Ordinate werden die addierten Gewichtsanteile angegeben. Auf der Abszisse erfolgt im allgemeinen eine logarithmische Teilung mit der Basis 10. Man erhält so für Lösse die bekannten charakteristischen Kurvenzüge.

Für viele Zwecke genügt allein die Ermittlung der Korngrößenzusammensetzung und ihre graphische Darstellung, so z. B. wenn festgestellt werden soll, ob es sich bei dem zu untersuchenden Substrat überhaupt um einen Löß handelt. Sehr oft steht man aber vor der Aufgabe, die Korngrößenzusammensetzung in einem mehrere Meter mächtigen Lößprofil zu ermitteln, um festzustellen, ob und wie sich die Körnung bzw. die Feinheit in der betreffenden Lößdecke ändert. Nur sehr selten können jedoch trotz der zahlreichen Körnungsanalysen genauere Angaben über die Änderung der Feinheit gemacht werden, denn die Fülle der Zahlen verwirrt und läßt keinen sicheren Schluf́s zu über den unter Umständen eingetretenen Wechsel der Körnung. Vor allem ist es nicht möglich, Angaben über das $\mathrm{M}$ a $ß$ der jeweiligen Schwankungen der Korngrößenzusammensetzung bzw. der F e in h e it zu machen. Auch aus der Summenkurve können solche quantitativen Angaben nicht entnommen werden. Die Beantwortung der Frage, ob von zwei Lössen der eine feiner ist als der andere, ist nur dann möglich, wenn eine der beiden Kurven in ihrer gesamten Länge oder zumindest in ihrem größten Teil (im Bereich der feineren Fraktionen) über der anderen verläuft.

Um Körnungsanalysen von Lössen besser miteinander vergleichen zu können, addieren manche Bearbeiter benachbarte Fraktionen und erhalten auf diese Weise weniger Zahlen. So ist z. B. W. FAULER (1936) verfahren, der die 3 feinsten Fraktionen addierte und die erhaltenen Werte für die Beurteilung des Feinheitsgrades zugrunde legte (vgl. Tab. 4). Man muß sich aber hierbei darüber im klaren sein, daß durch die Bildung von solchen Teilsummen und deren Vergleich niemals exakte Ergebnisse über die Anderung der Feinheit erzielt werden können, weil ja nur ein Teil der Korngruppen in Betracht gezogen wird. Wenn jedoch der Feinheitsgrad von Lössen miteinander verglichen wer.* den soll, so müssen in jedem Fall die Werte unter Berïcksichtigung a 11 e r Korngrupfen gewonnen werden. Erst dann kann von einem Feinheitsgrad gesprochen werden.

1) Früher wurden die Fraktionen meist folgendermaßen eingeteilt: $\langle 0,01,0,01-0,05,0,05-$ $0,1,0,1-0,2,0,2-0,5,0,5-1,0,1,0-2,0$ und $>2 \mathrm{~mm}$. 
Bei den vor über 10 Jahren durchgeführten Untersuchungen des Verfassers (E. SchöNHALS 1952) über den Zusammenhang zwischen Körnung und Kalkgehalt des Lösses wurde versucht, eine Methode zu finden, die es erlaubt, die Feinheit einer Probe in einer einzigen Zahl wiederzugeben. Wenn dies gelang, so war es möglich, aus der Kennzahl für den Feinheitsgrad (F) und aus dem Kalkgehalt (K) den spezifischen Kalkgehalt (Ks) zu berechnen (Ks $=\frac{F}{K}$; Näheres E. Schönhals 1952).

Eine solche Methode hat E. BLÜMEL (1933) für die Aufbereitungstechnik ausgearbeitet, um die unübersichtlichen Ergebnisse der Siebanalysen in Kennzahlen besser erfassen zu können. Eine Anwendung dieser sehr einfachen Methode mußte auch in der Lößforschung möglich sein, was auch durch die von hunderten von Körnungsanalysen ermittelten Kennzahlen bestätigt wurde. Seit dieser Zeit hat sich die Berechnung der Kennzahl bei den Untersuchungen des Verfassers als äußerst brauchbar erwiesen. Die Methode und ihre Anwendung sollen daher auf den folgenden Seiten näher beschrieben werden.

\section{Die Berechnung der Kennzahl des Feinheitsgrades}

Zur Erläuterung der Methode gehen wir zunächst von 3 einfachen Beispielen aus, die nicht den Löß betreffen. Die Substrate sollen nur aus Teilchen $\langle 1,0 \mathrm{~mm}$ bestehen. Der Kornspielraum sei gleich groß, nämlich $0,1 \mathrm{~mm}$. Die unterschiedliche Zusammensetzung der 3 Proben ist aus den Analysen 1 bis 3 zu ersehen (Tab. 1).

$$
\text { Tabelle } 1
$$

Die Körnung der Substrateder Anaiysen 1 bis 3

\begin{tabular}{|c|c|c|c|c|c|c|c|c|c|c|}
\hline & $\langle 0,1$ & $\begin{array}{l}0,1- \\
0,2\end{array}$ & $\begin{array}{l}0,2- \\
0,3\end{array}$ & $\begin{array}{l}0,3- \\
0,4\end{array}$ & $\begin{array}{l}0,4- \\
0,5\end{array}$ & $\begin{array}{l}0,5- \\
0,6\end{array}$ & $\begin{array}{l}0,6- \\
0,7\end{array}$ & $\begin{array}{l}0,7- \\
0,8\end{array}$ & $\begin{array}{l}0,8- \\
0,9\end{array}$ & $\begin{array}{l}0,9- \\
1,0 \mathrm{~mm}\end{array}$ \\
\hline Analyse 1 & 10 & 10 & 10 & 10 & 10 & 10 & 10 & 10 & 10 & $\begin{array}{l}10=100 \\
\text { Gew. } \%\end{array}$ \\
\hline Analyse 2 & 25 & 20 & 10 & 10 & 10 & 5 & 5 & 5 & 5 & $\begin{array}{r}5=100 \\
\text { Gew. } \%\end{array}$ \\
\hline Analyse 3 & 40 & 20 & 10 & 5 & 5 & 5 & 5 & 5 & 5 & $\begin{array}{r}0=100 \\
\text { Gew. } \%\end{array}$ \\
\hline
\end{tabular}

Bei der Analyse 1 sind alle 10 Fraktionen mit je 10 Gew. $\%$ /o beteiligt. Die Analysen 2 und 3 weisen diese gleichmäßige Körnung nicht mehr auf. Man sieht, daß das Substrat der Analyse 2 eine größere Feinheit hat als das der Analyse 1. Die Probe 3 ist noch feiner als das Substrat der Analyse 2. Die Feinheit nimmt also von Analyse 1 nach Analyse $3 \mathrm{zu}$. Mehr kann man zunächst nicht aussagen. Vor allem ist es nicht möglich, irgend eine Angabe zu machen, wie stark sich die einzelnen Proben in ihrer Feinheit unterscheiden.

Zur besseren Ubersicht werden die Zahlenwerte der 3 Analysen als Summenkurve graphisch aufgetragen. Wir erhalten dann die in Abb. 1 dargestellten Summenlinien. Da bei der Analyse 1 alle 10 Gewichtsanteile gleich groß sind, nämlich 10\%, bildet die Summenlinie eine Diagonale.

Die Kurve der Analyse 2 zeigt, da sie in den beiden ersten feinen Fraktionen höhere Gewichtsprozente aufweist, einen steileren Anstieg, läuft dann aber bis zur Korngruppe 0,4-0,5 mm mit der diagonalen Summenlinie parallel, um von da an in geradem Verlauf den Wert $100 \mathrm{zu}$ erreichen. Einen ähnlichen, aber im ersten Teil steileren Verlauf besitzt auch die Kurve der Analyse 3. Die Kurven der Analysen 2 und 3 liegen infolge ihrer größeren Feinheit links oberhalb der Linie 1. Proben, deren Kurven rechts unterhalb der Diagonale verlaufen würden, hätten demgegenüber eine gröbere Zusammensetzung als die Analyse 1. 


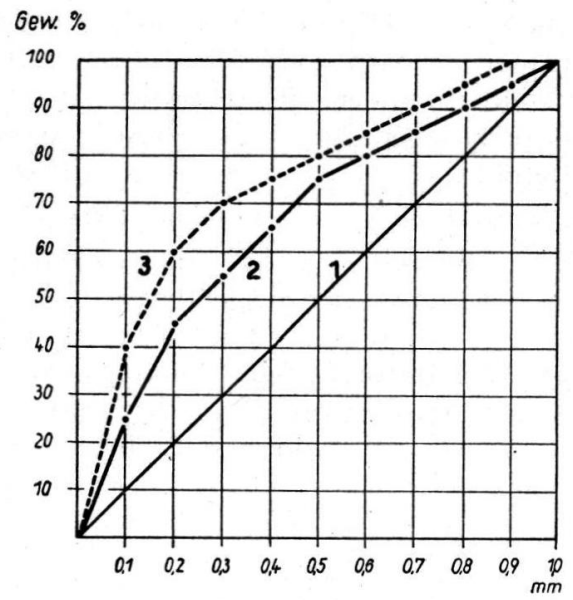

Abb. 1. Die Summenkurven der Analysen 1, 2 und 3.

Aus den drei Linienzügen geht hervor, daß der Unterschied in der Feinheit zwischen den Proben 1 und 2 wesentlich größer ist als zwischen 2 und 3. Irgend eine qu a n t i t a tive Angabe kann jedoch nicht gemacht werden. Will man dies erreichen, so ist es notwendig, die Flä che als Maß der Feinheit zugrunde zu legen und zwar diejenige, die von der Abszissenachse und der betreffenden Integralkurve umgrenzt wird. Diese Fläche wird dann mit einer maximalen Fläche, die eine Probe größter Feinheit repräsentiert, verglichen. Der prozentuale Anteil der von der jeweiligen Integralkurve umschlossenen Fläche an der Maximalfläche ergibt die Kennzahl des Feinheitsgrades.

Ein Substrat von größter Feinheit würde demnach durch eine Kurve dargestellt werden, die vom Nullpunkt senkrecht bis zur Ordinate 100 verläuft und von hier der oberen Begrenzung des Quadrates folgt. Es würde also das gesamte Quadrat erfaßt, das bei 10 Fraktionen und 100 Gewichtsprozenten 1000 Flächeneinheiten umschließt. Gehört dagegen das gesamte Substrat der gröbsten Fraktion an, so fällt die Kurve mit der Abszissenachse und der rechten Quadratseite zusammen. Der Flächeninhalt bzw. die Kennzahl ist demnach gleich Null. Um nun die einzelnen Flächengrößen als Prozentzahlen angeben zu können, dividiert man die Gesamtfläche durch die Zahl der unter-

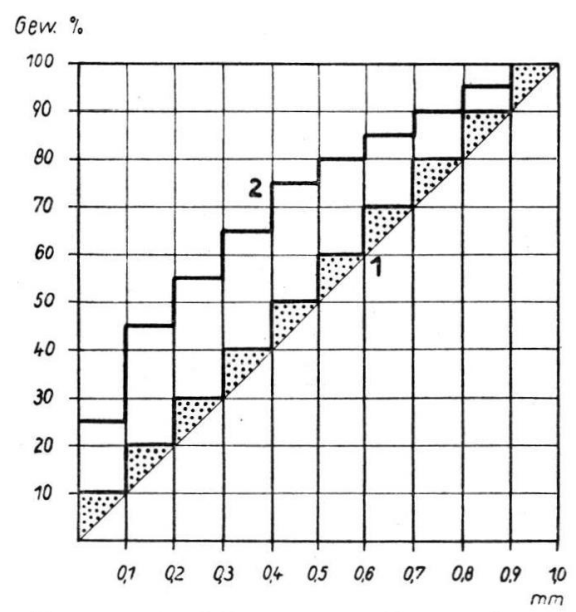

Abb. 2. Darstellung der addierten Gewichtsprozente der Analysen 1 und 2 als Rechtecke über den Kornklassen. 
schiedenen Fraktionen. In unserem Beispiel mïßte also 1000 durch 10 dividiert werden; man erhält dann die Kennzahl des Feinheitsgrades, nämlich $100 \%$. Mit zunehmender Feinheit steigen also die Kennzahlen an.

Die Ermittlung der Flächengröße könnte durch Planimetrieren oder Ausmessen erfolgen. Es ist aber auch möglich, die Größe der Fläche aus den einzelnen Gewichtsprozenten verhältnismäßig schnell zu berechnen. Errichtet man nämlich über den einzelnen Kornklassen, die in dem gewählten Beispiel alle 0,1 mm betragen, Rechtecke bis zur Höhe der jeweils addierten Gewichtsanteile, so entstehen an Stelle der einzelnen Summenlinien treppenförmige Linien (Abb. 2). Die zu den einzelnen Analysen gehörenden Gesamtflächen können dann durch Addition der einzelnen Rechtecke, die ja den addierten Gewichtsprozenten entsprechen, erhalten werden. Für die Analysen 1, 2 und 3 ist die Berechnung in Tabelle 2 durchgeführt.

Tabelle 2 .

Die Berechnung der Kennzahlen

Die addierten Gewichtsanteile betragen:

\begin{tabular}{lccc} 
& Analyse 1 & Analyse 2 & Analyse 3 \\
& 10 & 25 & 40 \\
& 20 & 45 & 60 \\
& 30 & 55 & 70 \\
& 40 & 65 & 75 \\
& 50 & 75 & 80 \\
& 60 & 80 & 85 \\
& 70 & 85 & 90 \\
Summe der addierten Gewichtsanteile: & 80 & 90 & 95 \\
Kennzahl: & 90 & 95 & 100 \\
\cline { 2 - 4 } & 100 & 100 & 100 \\
\hline
\end{tabular}

Die Summen der addierten Gewichtsanteile müssen noch durch 10 (Anzahl der Fraktionen) dividiert werden und man erhält dann die entsprechenden Kennzahlen (55,0, 71,5 und 79,5\%). Aus diesen Zahlen sind nun die Unterschiede der Feinheit viel besser und leichter zu erkennen als aus den Analysenergebnissen oder den Integralkurven.

Es sei an dieser Stelle noch erwähnt, warum die Kennziffer der Analyse 1, die ja eine mittlere Feinheit hat, nicht 50 sondern 55 beträgt. Die Ursache hierfür ist darin zu suchen, daß die Gesamtfläche um 10 rechtwinkelige Dreiecke von je 5 Flächeneinheiten zu groß ist (in Abb. 2 punktiert). Auch die beiden anderen Kennzahlen sind um $5 \% \mathrm{zu}$ hoch, weil die von den Integralkurven und den treppenförmigen Linienzügen gebildeten rechtwinkeligen Dreiecke ebenfalls eine Gesamtfläche von je 50 Einheiten umschließen. Dieser Nachteil läßt sich nicht vermeiden und ist auch nicht schwerwiegend, da es sich bei den Kennzahlen ja nicht um a bs ol u te Werte, sondern um Vergleichszahlen für Substrate mit einer ähnlichen Korngrößenzusammensetzung handelt. Diese Voraussetzung wird von dem Löß in idealer Weise erfüllt.

Es ist auch außerdem notwendig, immer dieselben Fraktionen zu wählen. In diesem Zusammenhang sei noch erwähnt, daß die Genauigkeit der Kennzahl mit der Anzahl der Fraktionen zunimmt. Verfasser benutzt die Unterteilung in 10 Fraktionen, was auch die Berechnung der Kennzahl etwas erleichtert. Es könnte nun an dieser Stelle eingewendet werden, daß die Fraktionen nicht gleich groß sind und dadurch auch ihr Einfluß auf die Höhe der Kennzahl nicht gleich ist (bei einer engen Korngruppe größer als bei einer weiten). Nach Berechnungen von BLÜMEL (1938) ergeben aber auch verschieden große Fraktionen durchaus noch vergleichsfähige Kennzahlen. 
Ein Vergleich von Kennzahlen ist also nur statthaft, wenn die angeführten Voraussetzungen erfüllt sind. Es sei auch noch darauf hingewiesen, daß sowohl die Vorbehandlung der Proben als auch die Untersuchungsmethode selbst immer dieselben sein müssen. Es ist daher anzustreben, bei der Erforschung des Lösses zukünftig nach einheitlichen Labor-Methoden zu arbeiten. Nur dann sind die Ergebnisse der mechanischen und chemischen Analysen (Kalkbestimmung) miteinander zu vergleichen.

III. Einige Beispiele für die Anwend.ung der Kennzah ${ }^{1}$ )

a) Die Änderung der Feinheit des Lösses im Profil

1. Schnell (1928) untersuchte die mechanische und chemische Zusammensetzung des Lösses in der Ziegelei Gebr. Schnell, Sprendlingen, Rheinhessen. Das Profil wurde bereits 1920 von HoHenstein beschrieben. Die Untersuchung der in einem Abstand von 0,5 m entnommenen Proben im Schöne'schen Schlämmapparat ergab für die Proben Nr. 4 bis 14 die in Tab. 3 angegebene Korngrößenzusammensetzung.

Ta belle 3

Korngrößenzusammensetzung des Lösses in der Zgl. Schnell, a m Bahnhof Sprendlingen (Rheinhessen)

\begin{tabular}{c|ccccc|c|c}
\hline $\begin{array}{c}\text { Nr. } \\
\text { der } \\
\text { Probe }\end{array}$ & \multicolumn{5}{c|}{ Korngrößenzusammensetzung in mm } & \multirow{2}{*}{ Kennzahlen } \\
\hline 4 & $<0,01$ & $0,01-0,05$ & $0,05-0,1$ & $0,1-0,2$ & $>0,2$ & \\
\hline 5 & 11,7 & 41,4 & 30,5 & 12,5 & 3,9 & 68,7 \\
6 & 11,3 & 50,7 & 25,1 & 9,1 & 3,8 & 71,3 \\
7 & 11,1 & 55,1 & 23,2 & 7,8 & 2,8 & 72,8 \\
8 & 14,2 & 54,3 & 22,8 & 7,1 & 1,6 & 74,5 \\
9 & 13,0 & 53,2 & 22,8 & 7,5 & 3,5 & 72,9 \\
10 & 9,6 & 57,8 & 22,9 & 7,4 & 2,3 & 73,0 \\
11 & 11,4 & 54,5 & 26,6 & 6,0 & 1,5 & 73,7 \\
12 & 11,2 & 63,9 & 14,8 & 6,8 & 3,3 & 74,6 \\
13 & 10,8 & 71,6 & 12,8 & 3,9 & 0,9 & 76,9 \\
14 & 10,6 & 72,3 & 11,6 & 2,6 & 2,9 & 77,0 \\
& 11,7 & 67,6 & 14,0 & 5,6 & 1,1 & 76,6
\end{tabular}

Die aus den 5 Fraktionen berechneten Kennzahlen des Feinheitsgrades sind in Tabelle 3 rechts aufgeführt. Zur besseren Übersicht wurden die Kennzahlen graphisch auf-

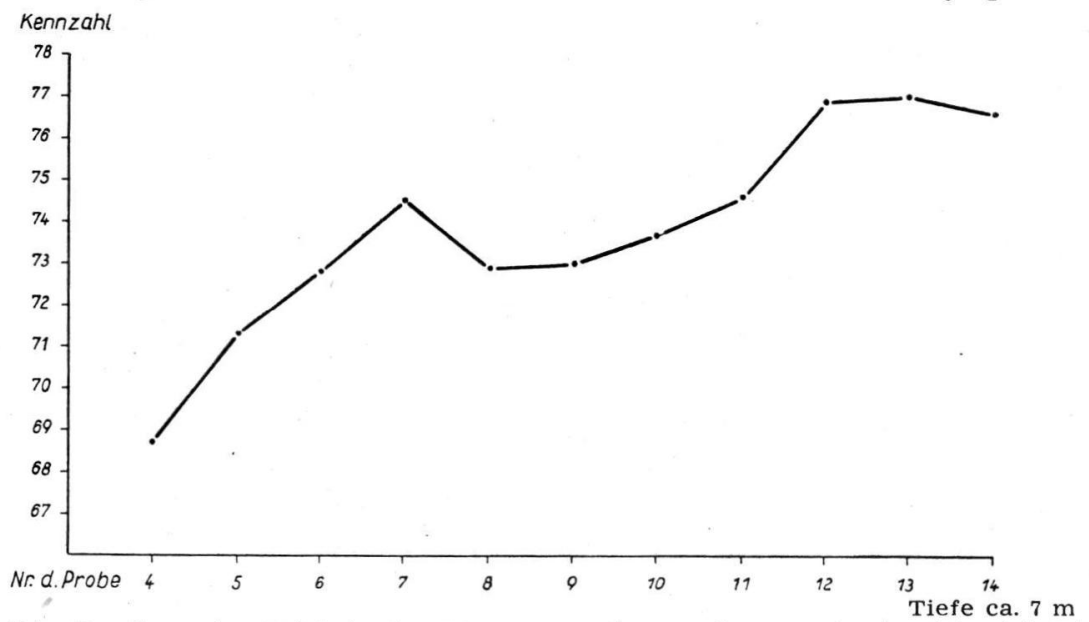

Abb. 3. Die Zunahme der Feinheit des Lösses von oben nach unten in der Ziegelei Schnell, Sprendlingen.

1) Die Unterlagen der folgenden 7 Beispiele sind bereits veröffentlichten Arbeiten entnommen. Es besteht daher die Möglichkeit, Einzelheiten über die benutzten Beobachtungen nachzulesen. 
getragen (Abb. 3). Schon auf den ersten Blick erkennt man im oberen Teil des Profils die fast gleichmäßige Zunahme der Feinheit nach der Tiefe. Die Proben 8 und 9 sind aber wieder etwas gröber als Probe 7; von Probe 10 an nimmt jedoch der Feinheitsgrad wieder zu, um sich bei den 3 letzten Proben kaum noch zu ändern. Der Kurvenzug läßt also eine deutliche $Z_{w}$ eiteilung des Profils erkennen, was aus den 55 Einzelwerten nicht hervorgeht. Erst die 11 Kennzahlen bzw. ihre Eintragung in das Koordinatenkreuz sind geeignet, die Änderungen des Feinheitsgrades schnell vor Augen zu führen. Die Feinheit des Lösses nimmt also sowohl im oberen als auch im tieferen Profilabschnitt von oben nach unten zu.

2. Als nächstes Beispiel sei das von W. FAulen (1936) untersuchte Profil von Fautenbach (Eichberg) kurz besprochen. Nach W. FAuler gliedert sich der etwa $5 \mathrm{~m}$ mächtige Löß in einen Jüngeren Löß I $(1,5 \mathrm{~m})$ und einen Jüngeren Löß II, der etwa 2,7 m mächtig ist. Auf dem Jüngeren Löß I ist die $0,8 \mathrm{~m}$ mächtige Verlehmungsrinde erhalten geblieben. Aus der gesamten Lößfolge wurden von W. FAuler in Abständen von 30 bis $50 \mathrm{~cm}$ insgesamt 10 Proben entnommen, deren Korngrößenzusammensetzung aus Tab. $4 \mathrm{zu}$ ersehen ist (Methode Atterberg).

Tabelle 4

Korngrößenzusammensetzung und Kennzahlen des Lößprofils von Fa uten bach. Nach W. Fauler (1936) Taf. C, Tab. 19.

\begin{tabular}{|c|c|c|c|c|c|c|c|}
\hline \multirow{3}{*}{$\begin{array}{c}\text { Stratigraphische } \\
\text { Bezeichnung }\end{array}$} & \multirow{3}{*}{$\begin{array}{l}\text { Nr. } \\
\text { der } \\
\text { Probe }\end{array}$} & \multicolumn{5}{|c|}{ Korngrößenzusammenseţung in $\mathrm{mm}$} & \multirow{3}{*}{ Kennzahlen } \\
\hline & & $<0,008$ & $\begin{array}{l}0,008 \text { - } \\
0,03\end{array}$ & $\begin{array}{l}0,03- \\
0,067\end{array}$ & $>0,067$ & $<0,03$ & \\
\hline & & $\mathrm{I}+\mathrm{II}$ & III & IV & $\mathrm{V}$ & $\underset{\text { III }}{\mathrm{I}}+$ & \\
\hline \multirow{4}{*}{$\begin{array}{l}\text { Jüngerer } \\
\text { Löß II }\end{array}$} & 72 & 6,89 & 30,62 & 47,63 & 14,86 & 37,51 & 57,4 \\
\hline & 71 & 7,49 & 32,08 & 43,49 & 16,94 & 39,57 & 57,5 \\
\hline & 70 & 5,65 & 27,45 & 55,65 & 11,25 & 33,10 & 56,9 \\
\hline & 69 & 5,24 & 28,08 & 56,11 & 10,57 & 33,32 & 57,0 \\
\hline \multirow{3}{*}{$\begin{array}{l}\text { Lößlehm } \\
\text { (aus Jüngerem } \\
\text { Löß I) }\end{array}$} & 68 & 14,26 & 38,37 & 37,30 & 10,07 & 52,63 & 64,2 \\
\hline & 67 & 22,10 & 27,70 & 34,09 & 16,11 & 49,80 & 64,0 \\
\hline & 66 & 13,65 & 28,55 & 46,28 & 11,52 & 42,20 & 61,1 \\
\hline \multirow{3}{*}{$\begin{array}{l}\text { Jüngerer } \\
\text { Löß I }\end{array}$} & 65 & 10,67 & 27,69 & 46,71 & 14,93 & 38,36 & 58,5 \\
\hline & 64 & 7,62 & 28,15 & 55,33 & 8,90 & 35,77 & 58,6 \\
\hline & 63 & 4,37 & 18,45 & 64,99 & 12,19 & 22,32 & 53,8 \\
\hline
\end{tabular}

Um die Änderungen in der Körnung erfassen zu können, zählte W. FAULER die Gewichtsprozente der feinsten Fraktionen I, II und III jeweils zusammen und schloß aus diesen Werten auf die Feinheit. Dieses Verfahren ist - wie früher schon angedeutet wurde - mit einem Mangel behaftet, denn erstens ist die gewählte Grenze $(0,03 \mathrm{~mm})$ eine willkürliche und zweitens darf man zur Beurteilung des Feinheitsgrades einer Probe nicht nur die 3 feinsten Fraktionen benutzen. Es müssen vielmehr a $11 \mathrm{e}$ Kornklassen herangezogen werden. Ist dies nicht der Fall, so kann auch nicht von der Feinheit einer Probe gesprochen werden. Die Ermittlung der Kennzahl bietet nun die Möglichkeit, unter Berücksichtigung aller Fraktionen ein Maß für die Feinheit zu erhalten.

Trägt man nun die aus den 40 Prozentzahlen errechneten 10 Kennzahlen wiederum graphisch auf (Abb. 4), so treten die Körnungsunterschiede zwischen den einzelnen Proben in dem Verlauf einer einzigen Kurve klar hervor. 


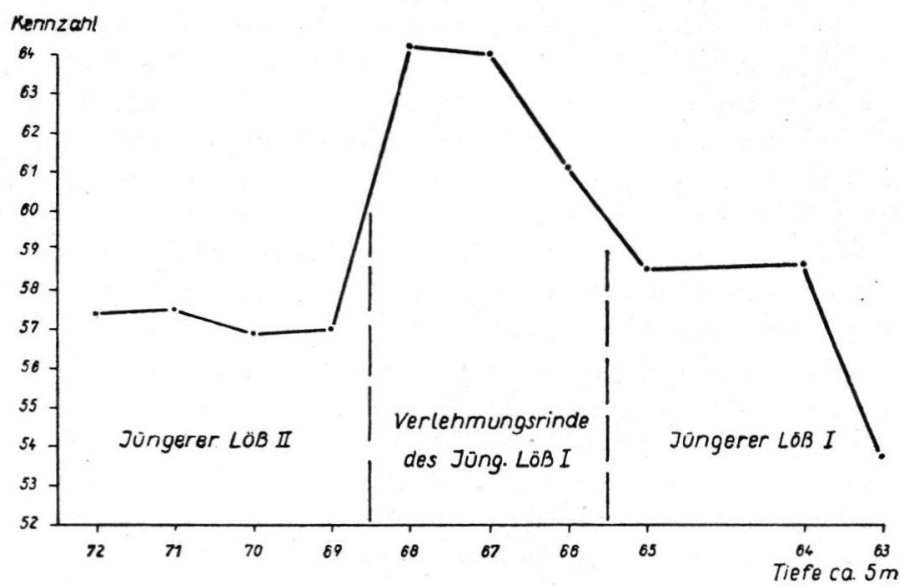

Abb. 4. Die Feinheitsunterschiede im Lößprofil von Fautenbach.

Zwei Besonderheiten des Lößprofils sind auf den ersten Blick festzustellen. Das ist einmal die wesentlich feinere Beschaffenheit der Verlehmungszone und zweitens die unterschiedliche Feinheit der beiden Lösse. Man erkennt, daß der Jüngere Löß II nicht mehr die Feinheit erreicht wie der Jüngere Löß I.

W. FAuler hat darauf hingewiesen, daß der unverschwemmte Altere und Jüngere Löß nach oben stetig feiner werden. Er stützte sich allerdings bei dieser Behauptung nur auf die feinsten Fraktionen (I + II + III). Vergleicht man jedoch die in Tab. 4 angegebenen Kennzahlen sowie diejenigen von den hier nicht mitgeteilten Körnungsanalysen, so ergibt sich, daß diese Regel nicht immer zutrifft. An dieser Stelle muß auch erwähnt werden, daß F. ZINK (1940) durch seine Untersuchungen die Angaben W. FAULER's nicht bestätigen konnte.

3. Als drittes Beispiel sei das von F. ZINK (1940) bearbeitete Pleistozänprofil von Murg (Hochrhein) erläutert. Nach F. Zink gliedert sich das Profil folgendermaßen: Uber der verwitterten Riß I-Moräne folgt der Altere Löß des Riß II mit einer etwa $3 \mathrm{~m}$ mächtigen Verlehmungsdecke (Riß II-Würm I-Interglazial). Der obere etwa $6 \mathrm{~m}$ mächtige Teil wird vermutlich von zwei Jüngeren Lössen gebildet, von denen der obere zwei Meter tief verlehmt ist.

Aus der gesamten Schichtenfolge wurden von F. ZINK 28 Proben entnommen und nach der Methode Atterberg untersucht. Die Ergebnisse der Körnungsanalysen sind in Tab. 5 zusammengestellt. F. ZINK unterschied die gleichen 5 Fraktionen wie W. Fauler; die Vorbehandlung der Proben war jedoch nicht dieselbe. Ein exakter Vergleich der Kennzahlen ist daher nicht möglich.

Schon bei einer ersten Durchsicht der 112 Analysenwerte ist ein recht beträchtlicher. Wechsel in der Körnung zu beobachten. Trotzdem erkennt man, daß im höheren Teil der fossilen Verwitterungsdecke die Feinheit nach oben hin zunimmt, worauf auch F. ZINK (S. 36) hinweist. Eine Zunahme der Feinheit der Lösse von unten nach oben konnte F. ZINK im Gegensatz zu W. Fauler jedoch nicht nachweisen.

Ein noch viel deutlicheres Bild von den Änderungen des Feinheitsgrades erhalten wir auch hier durch die Berechnung der 28 Kennzahlen und ihre graphische Darstellung (Abb. 5). Sofort erkennt man, daß die unteren $4 \mathrm{~m}$ eine wechselnde Zusammensetzung haben und erst im oberen Teil des Lehmes die Feinheit stetig zunimmt. Die größte Feinheit besitzt der oberste Teil des Lehms, was wahrscheinlich eine Folge intensiver Verwitterung ist. Der über dem Lehm liegende Jüngere Löß weist wiederum einen 
ziemlich raschen Körnungswechsel auf, doch beobachtet man, daß mit zunehmender Höhe im Profil die Feinheit abnimmt.

$$
\text { Tabelle } 5
$$

Korngrößenzusammensetzung und Kennzahlen des Lösses in der Ziegelei Murg (Profil I an der Westwand). Nach F. ZINK (1940).

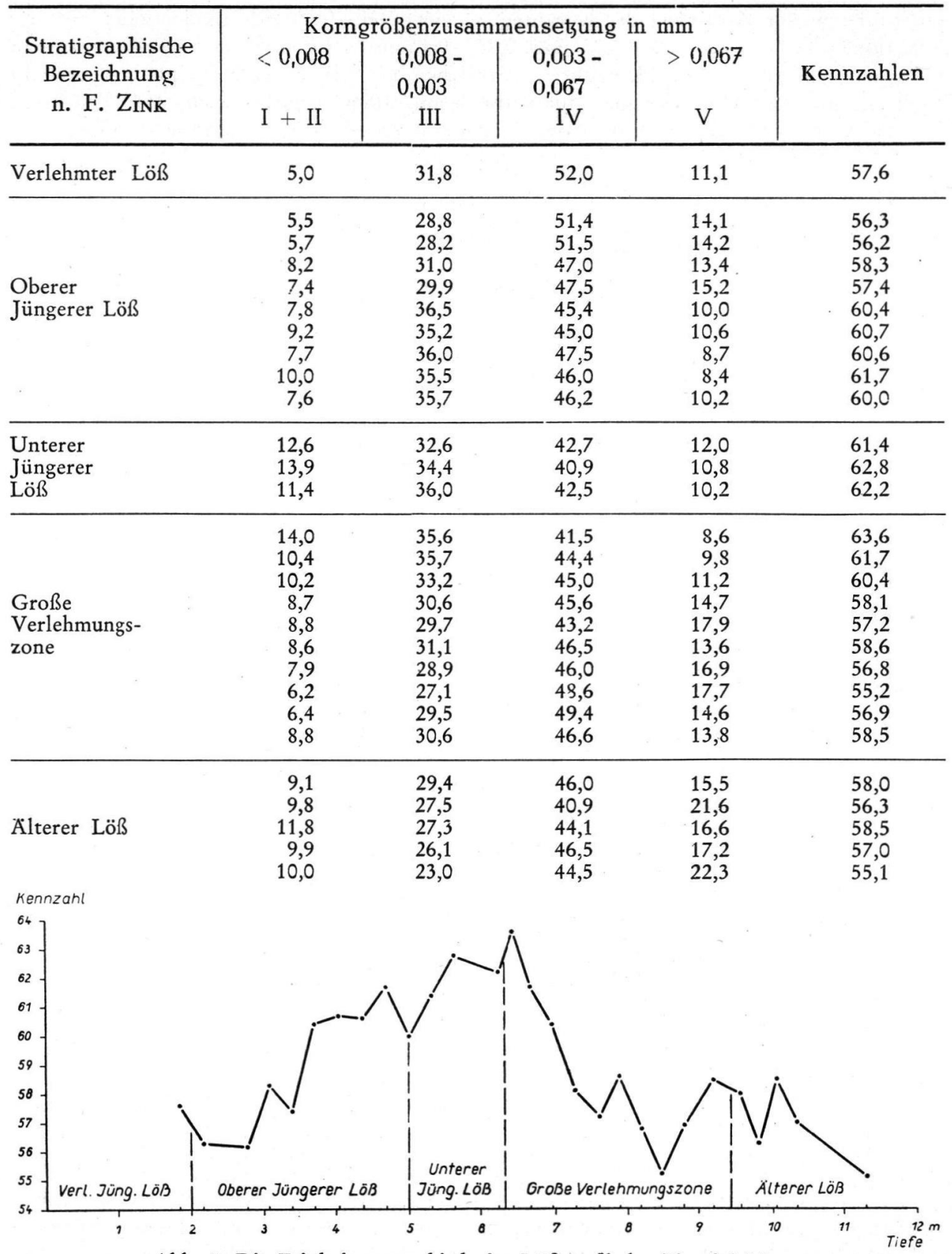

Abb. 5. Die Feinheitsunterschiede im Lößprofil der Ziegelei Murg. 
4. Recht aufschlußreich sind auch die Kennzahlen von einem Flottsandprofil aus der istlichen Mark Brandenburg, das Br. DAMmer (1941) näher beschrieben hat. Die Entnahmestelle der Proben lag auf dem Blatt Lagow und zwar an der Straße von Neulagow nach der Försterei Langenpfuhl. Aus der etwa 1,2 m mächtigen Flottsanddecke wurden in Abständen von $10 \mathrm{~cm} 12$ Proben entnommen. Die Ergebnisse der Körnungsanalysen finden sich in Tab. 6. Aus den insgesamt 108 Zahlen ist jedoch nur schwer eit. Bild von der Änderung der Körnung zu gewinnen. Lediglich an der Zunahme der Fraktionen $0,01-0,02,0,02-0,05$ und $0,05-0,1 \mathrm{~mm}$ zwischen 20 und $70 \mathrm{~cm}$ kann man erkennen, daß in diesen Horizonten wahrscheinlich die größten Feinheiten erreicht werden, eine Annahme, die auch durch die Kennzahlen bestätigt wird. Viel deutlicher spiegelt aber die Kurve in Abb. 6 den Körnungswechsel der Flottsanddecke wider.

Tabelle 6

Korngrößenzusammensetzung und Kennzahlen des Flottsandes an der Straße Neulagow-Försterei Langenpfuhl, Bl. Lagow . Nach B. Dammer 1941, S. 194.

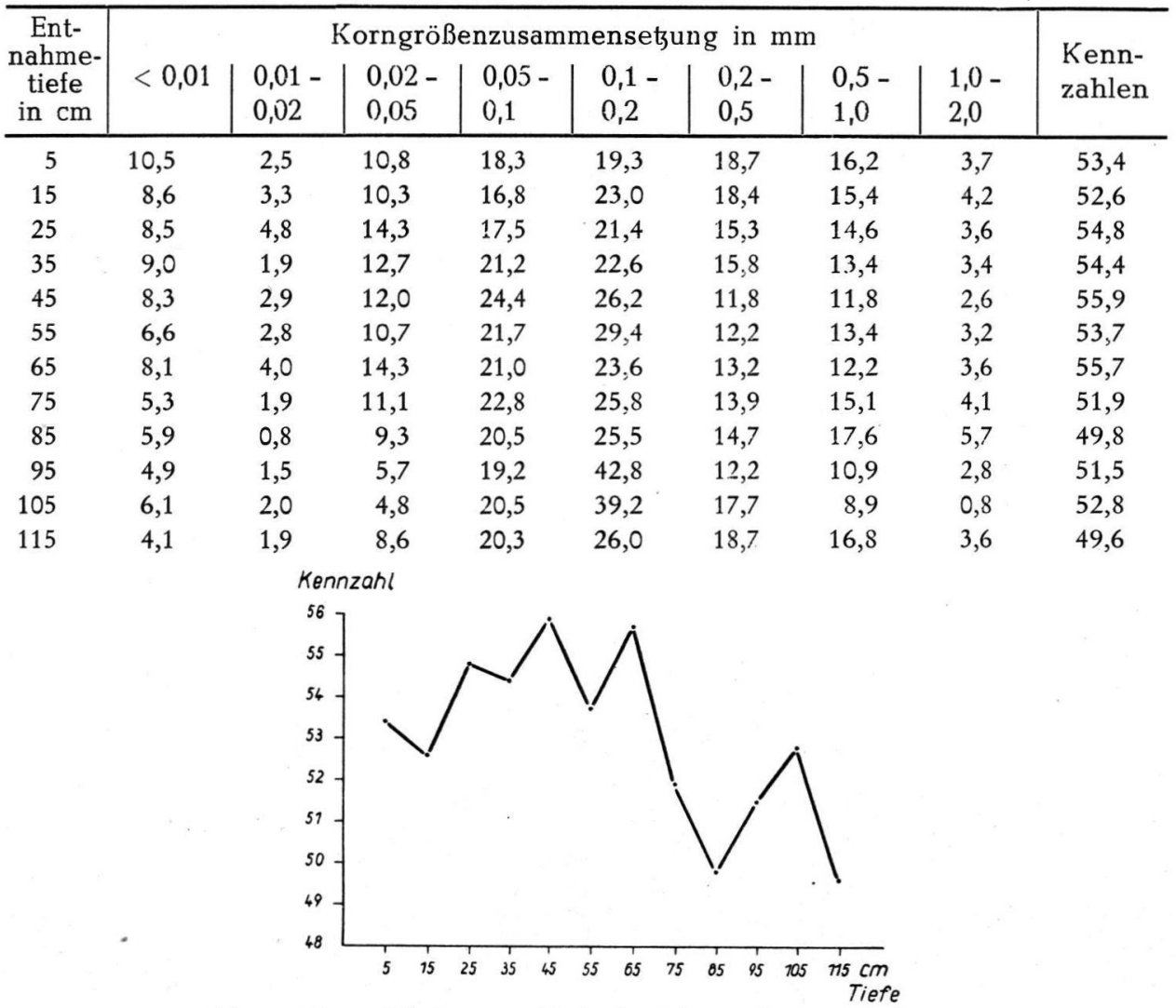

Abb. 6. Die Feinheitsunterschiede im Flottsand von Neulagow.

b) Die Anderung der Feinheit des Lösses in horizontaler Richtung

Genau wie bei den anderen Sedimentgesteinen können auch beim Löß verschiedene Faziesbereiche unterschieden werden, wie dies vor kurzem an einem näher untersuchten Gebiet dargelegt wurde (E. Schönhals 1953). Zur Abgrenzung der einzelnen Fazies- 
bereiche sind neben der feinstratigraphischen Aufnahme sämtlicher Aufschlüsse zahlreiche Körnungsanalysen erforderlich. Bei dem Vergleich des dann meist sehr umfangreichen Zahlenmaterials gelangt man jedoch hinsichtlich der Feinheit nur sehr selten zu eindeutigen Ergebnissen. Eine wesentlich bessere Übersicht ermöglichen auch hierbei die Kennzahlen. Außerdem erlauben sie erst, die Beziehungen zwischen dem Feinheitsgrad und der Entfernung vom Auswehungsgebiet zu erkennen und graphisch darzustellen. Es erübrigt sich von jetzt an, nur bestimmte Fraktionen für sich allein in Abhängigkeit von der Entfernung aufzutragen (vgl. Sмiтн 1942).

Die Brauchbarkeit der Kennzahlen bei solchen Untersuchungen sei nun an 3 Beispielen näher erläutert.

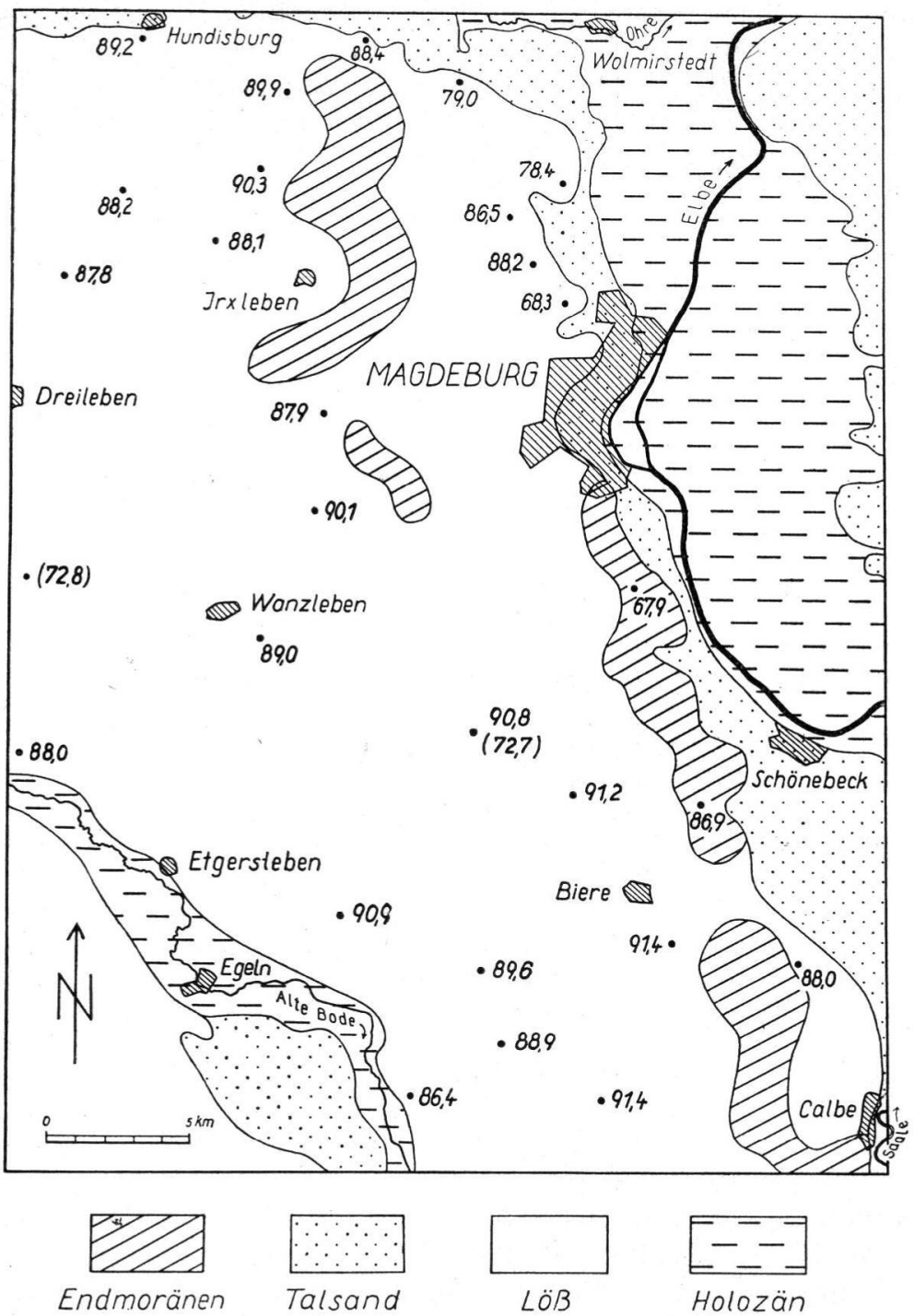

Abb. 7. Kennziffern des Feinheitsgrades von Lössen aus der Magdeburger Börde. Die eingeklammerten Kennziffern beziehen sich auf Sandbänder im Löß. 
1. In einer früheren Arbeit (E. Sснönhals 1952) wurde bereits auf die beachtlichen Unterschiede in der Körnung des Lösses der Magdeburger Börde hingewiesen. Wie aus den in der genannten Arbeit veröffentlichten mechanischen Analysen hervorgeht, kommen Grob-, Normal- und Feinlösse vor. Die gröberen Lösse liegen am Ostrand der Börde, was auf die Einwehung von gröberen Teilchen aus dem Elbtal zurückzuführen ist. Das gleiche beobachtet man auch verschiedentlich am Bodetal und im $\mathrm{N}$ an der Ohre. Nach Westen hin wird dann der Löß rasch feiner. Nur ab und zu treten in dem geschlossenen Lößgebiet noch einzelne Sandlagen auf, die auf lokalen Einwehungen von älterem pleistozänem Sand beruhen. An Hand von 28 Körnungsanalysen aus dem Gebiet zwischen Bode, Elbe und Ohre wurde s. Zt. versucht, eine erste allgemeine Übersicht über die mechanische Zusammensetzung des Bördelösses zu gewinnen. Die Analysen wurden in zusammengefaßter Form 1952 veröffentlicht. Von einer nochmaligen Wiedergabe der Ergebnisse wird daher abgesehen.

Viel besser als aus den 196 Einzelwerten der Körnungsanalysen sind die oben schon skizzierten Feinheitsunterschiede aus den 28 Kennzahlen zu ersehen, die an den Entnahmestellen eingetragen sind (Abb. 7). Auffallend ist, daß sich die feinsten Lösse unmittelbar westlich der Endmoränen oder in nicht allzu großer Entfernung von ihnen finden. Diese Erscheinung hängt vielleicht damit zusammen, daß die gröberen, in Bodennähe verwehten Teilchen durch die im allgemeinen 80 bis $125 \mathrm{~m}$ hohen Endmoränenwälle weitgehend zurückgehalten wurden.

Durch eine Vermehrung der Beobachtungsstellen und der Proben in den einzelnen Profilen wäre es wohl auch möglich, Lin i e n g l e i c h e r F e in h e i t zu konstruieren, die dann ein noch besseres Bild von den regionalen Unterschieden der Körnung vermittelten.

2. In einer kurzen Bemerkung zu einem Vortrag von H. BredDin über „Löß, Flug.sand und Niederterrasse im Niederrheingebiet" weist R. GrahmanN (1927) auf die Wechselbeziehungen zwischen Löß und Flugsand (Decksand) im ehemaligen Freistaat Sachsen hin (vgl. auch R. Grahmann 1932). Um nachzuweisen, daß zwischen dem 15 bis $20 \mathrm{~km}$ breiten und bis $1,5 \mathrm{~m}$ mächtig werdenden Flugsandstreifen im $\mathrm{N}$ und dem Löß im S eine Vermischung von Flugsand und Löß stattfindet, teilt R. Grahmann von dem Blatt Oschatz die in Tab. 7 angegebenen 4 Schlämmanalysen mit.

Tabelle 7

Korngrößenzusammensetzung und Kennzahlen von Lössen a us $\mathrm{S}$ a chsen

\begin{tabular}{|c|c|c|c|c|c|c|c|c|c|}
\hline $\begin{array}{c}\text { Nr. } \\
\text { der } \\
\text { Probe }\end{array}$ & $<0,01$ & $|0,01-0,05|$ & $\begin{array}{l}\text { Corngröße } \\
0,05-0,01 \mid\end{array}$ & $|0,1-0,2|$ & $|0,2-0,5|$ & $\begin{array}{l}\mathrm{g} \text { in } \mathrm{mm} \\
0,5-1,0\end{array}$ & $1,0-2,0$ & $>2,0$ & $\begin{array}{l}\text { Kenn- } \\
\text { zahlen }\end{array}$ \\
\hline 37 & 7,0 & $-14,4$ & 5,0 & 13,6 & 48,7 & 10,6 & 0,5 & 0,2 & 60,4 \\
\hline 32 & 17,2 & 29,5 & 7,2 & 17,0 & 21,6 & 4,7 & 1,9 & 0,9 & 71,5 \\
\hline 48 & 21,9 & 40,8 & 8,7 & 7,6 & $13, \mathrm{~s}$ & 4,7 & 1,8 & 0,7 & 78,0 \\
\hline $11 b$ & 20,5 & 63,6 & 7,5 & 3,4 & 3,1 & 1,0 & 0,3 & 0,6 & 86,0 \\
\hline
\end{tabular}

$\mathrm{H}$ e rk u n f t : Nr. 37 nordöstlich von Klingenhain; Nr. 32 nördlich von Sörnewitz;

Nr. 48 nordwestlich von Oschatz; Nr. 11b Vorwerk Weinhaus bei Borna.

Die von $\mathrm{N}$ nach $\mathrm{S}$ angeordneten Entnahmestellen liegen je etwa $3 \mathrm{~km}$ auseinander. Aus den Analysen ist zu ersehen, daß die für Flugsand typische Fraktion 0,2-0,5 mm nach $S$ rasch abnimmt, die für Löß charakteristische Korngruppe 0,01-0,05 mm aber zunimmt. Welche qua $\mathrm{t}$ it a t iven Unterschiede in der Feinheit zwischen den 4 Proben bestehen, geht jedoch aus der doch recht übersichtlichen Zahlentafel nicht hervor. 
Dies erlauben erst die 4 Kennzahlen. Noch eindeutiger sind die Zusammenhänge zwischen dem Feinheitsgrad und der Lage der Entnahmestellen aus der graphischen Darstellung zu erkennen (Abb. 8). Die Feinheit nimmt also mit dem Logarithmus der Entfernung vom Auswehungsgebiet zu, eine Beziehung, die das nächste Beispiel noch besser hervortreten läßt.

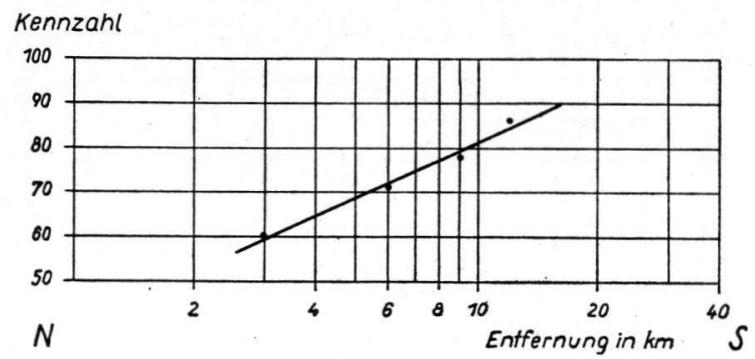

Abb. 8. Beziehung zwischen dem Feinheitsgrad und dem Logarithmus der Entfernung vom Auswehungsgebiet (Blatt Oschatz, Sachsen).

3. G. D. SMITH (1942) konnte an Hand einer Übersichtskarte der Lößmächtigkeit in Illinois zeigen ${ }^{1}$ ), daß die Mächtigkeit vom Mississippi- und Illinoistal nach $\mathrm{O}$ abnimmt. Während beispielsweise am Illinois River im County Mason die Mächtigkeit über $7 \mathrm{~m}$ beträgt, werden etwa 90 Meilen weiter südöstlich nur noch $0,5-1,0 \mathrm{~m}$ erreicht. In der gleichen Richtung nimmt die Feinheit des Lösses zu, wie G. D. SMITH durch Korngrößenanalysen nachweisen konnte. Die Proben wurden aus dem Peorian-Löß entlang von zwei in NW-SO-Richtung verlaufenden Profillinien entnommen (vgl. P. WoLDStedt 1954, S. 180). Auf die etwa $65 \mathrm{~km}$ lange Profillinie 1 entfallen 11 Proben, deren mechanischer Aufbau aus Tab. 8 zu ersehen ist. Man erkennt, daß die Gewichtsprozente der feinsten Fraktionen (bis $0,02 \mathrm{~mm}$ ) im allgemeinen nach $\mathrm{SO}$ ansteigen, die beiden gröberen Korngruppen $(0,03-0,04$ und $0,04-0,05)$ dagegen abnehmen. Die Fraktion $0,02-0,03 \mathrm{~mm}$ nimmt zuerst zu und dann ab. Der Löß wird also mit Zunahme der

Tabelle 8

Die Körnung des Lösses entlang eines NW-SO-Profils von dem Sangamon River durch die Counties Menard, Sangamon, Christian und Shelby (nach G. D. Sмith 1942).

\begin{tabular}{|c|c|c|c|c|c|c|c|c|}
\hline \multirow{2}{*}{$\begin{array}{l}\text { Lfd. } \\
\text { Nr. }\end{array}$} & \multirow{2}{*}{$\begin{array}{l}\text { Entfernung vom } \\
\text { Auswehungsgebiet } \\
\text { in Meilen }\end{array}$} & \multicolumn{6}{|c|}{ Korngrößenzusammenseţung in $\mathrm{mm}$} & \multirow{2}{*}{$\begin{array}{l}\text { Kenn- } \\
\text { zahlen }\end{array}$} \\
\hline & & $<0,001$ & $\left|\begin{array}{l}0,001- \\
0,01\end{array}\right|$ & $\begin{array}{l}0,01- \\
0,02\end{array}$ & $\begin{array}{l}0,02- \\
0,03\end{array}$ & $\begin{array}{l}0,03- \\
0,04\end{array}$ & $\begin{array}{l}0,04- \\
0,05\end{array}$ & \\
\hline 1 & 0,6 & 2,2 & 3,9 & 9,0 & 15,5 & 50,7 & 18,7 & 39,2 \\
\hline 2 & 1,5 & 3,4 & 6,3 & 9,4 & 14,1 & 45,3 & 21,5 & 40,7 \\
\hline 3 & 3,8 & 7,5 & 6,8 & 19,9 & 32,9 & 31,7 & 0,8 & 53,6 \\
\hline 4 & 4,5 & 6,1 & 10,9 & 20,8 & 24,6 & 34,9 & 2,7 & 53,4 \\
\hline 5 & 9,3 & 4,8 & 9,9 & 23,6 & 30,1 & 30,9 & 0,7 & 54,2 \\
\hline 6 & 9,3 & 5,3 & 11,5 & 21,0 & 27,7 & 33,1 & 1,4 & 54,0 \\
\hline 7 & 14,7 & 7,2 & 11,6 & 23,2 & 30,0 & 27,0 & 1,0 & 56,5 \\
\hline 8 & 19,6 & 7,2 & 13,7 & 25,2 & 27,2 & 26,1 & 0,6 & 57,8 \\
\hline 9 & 24,2 & 6,8 & 15,8 & 27,8 & 24,6 & 24,0 & 1,0 & 59,0 \\
\hline 10 & 42,0 & 11,3 & 18,0 & 31,7 & 21,5 & 16,6 & 0,9 & 63,9 \\
\hline 11 & 65,0 & 8,7 & 24,6 & 31,9 & 18,6 & 14,5 & 1,7 & 64,9 \\
\hline
\end{tabular}

1) Die Karte befindet sich auch als Schwarz-Weiß-Darstellung bei P. WóLDSTEDt 1954. 


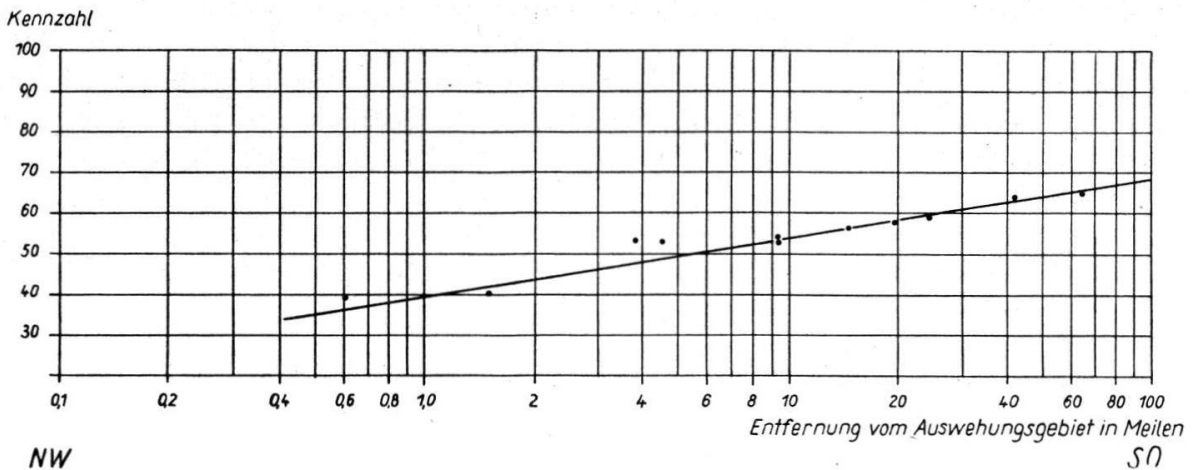

Abb. 9. Beziehung zwischen dem Feinheitsgrad und dem Logarithmus der Entfernung vom Auswehungsgebiet (Illinois).

Entfernung vom Auswehungsgebiet feiner. Die Zusammenhänge werden von G. D.

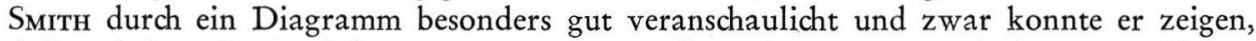
daß zwischen der Fraktion $0,01-0,02 \mathrm{~mm}$ und $0,03-0,05 \mathrm{~mm}$ und dem Logarithmus der Entfernung lineare Beziehungen bestehen. Da G. D. SMITH jedoch nur die Beziehungen zwischen einzelnen Fraktionen und der Entfernung ermitteln konnte, ist aus seinen Tabellen und graphischen Darstellungen nicht $\mathrm{zu}$ entnehmen, in welchem $\mathrm{Ma} ß \mathrm{e}$ die Gesamtfeinheit des Lösses von NW nach SO zunimmt. Dies ermöglichen erst die Kennzahlen des Feinheitsgrades, die in Tabelle 8 angegeben sind. Tragen wir die Kennzahlen zusammen mit dem Logarithmus der Entfernung in ein Koordinatenkreuz ein, so sehen wir, daß der Feinheitsgrad linear ansteigt (Abb. 9). In der Nähe des Auswehungsgebietes nimmt die Feinheit des Lösses wesentlich schneller zu als in größerer Entfernung, was aus Abb. 10 noch besser hervorgeht. Im Bereich der ersten 3 Meilen hat also bereits die Hauptseigerung des angewehten Materials stattgefunden. Von dieser Entfernung an nimmt die Feinheit zunächst noch etwas stärker, dann aber nur noch sehr wenig zu. So beträgt die Zunahme des Feinheitsgrades zwischen 3 und 20 Meilen etwa 5\%, zwischen 20 und 40 Meilen $3 \%$, die nächsten 40 Meilen 2\% und von 80-100 Meilen nur noch $1 \%$. Der Löß hat also in einer Entfernung von 80 Meilen praktisch seine größte Feinheit erreicht. Weiter östlich besitzt er eine fast gleichbleibende Körnung, sofern lokal keine Einwehungen von Fremdmaterial erfolgten. Der Zunahme des Feinheitsgrades entsprechend müßte daher in dem etwa 3 Meilen breiten Randgebiet auch die größte Mächtigkeit angetroffen werden, was auch aus den Messungen und Diagrammen von G. D. SMITH hervorgeht. So nimmt die Mächtigkeit des Lösses entlang der Profillinie 1 und im benachbar-

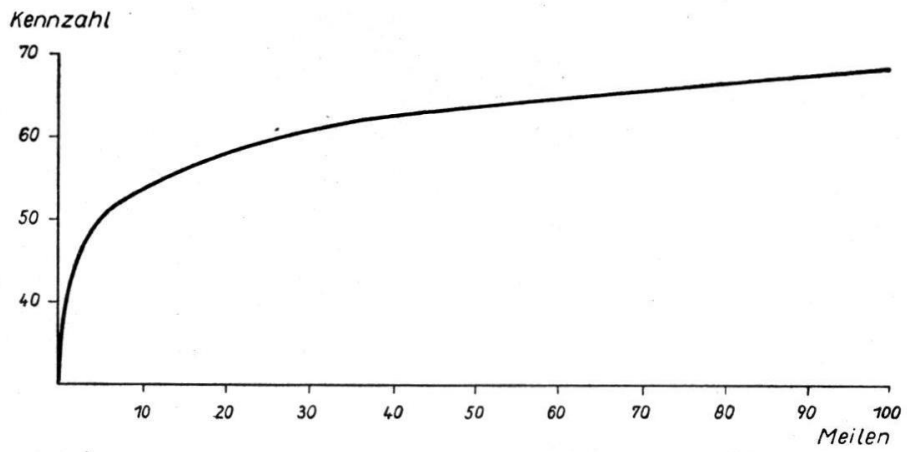

Abb. 10. Die Zunahme der Feinheit mit der Entfernung vom Auswehungsgebier. 
ten County Cass von 0,2 bis etwa 3 Meilen von $27,5 \mathrm{~m}$ auf etwa $6 \mathrm{~m}$ ab, während von 3 bis 50 Meilen die Abnahme nur etwas mehr als $4 \mathrm{~m}$ beträgt.

Auf weitere Beziehungen zwischen dem Feinheitsgrad, der Mächtigkeit, dem Kalkgehalt und der Entfernung vom Ursprungsgebiet kann an dieser Stelle nicht eingegangen werden. Der Zweck dieses Aufsatzes sollte auch nur sein, an einigen Beispielen den Wert der Kennzahlen für die Lößforschung aufzuzeigen.

\section{Schriftenverzeichnis}

BEHR, J. \& KöHLER, R.: Beitrag zur praktischen Auswertung der Bodenanalyse. - Mitt. Labor. d. preuß. geol. L.-A., H. 11, S. 15-34, Berlin 1930.

BLÜMEL, E.: Kennziffern für Siebanalysen und andere Zahlentafeln bei der Braunkohlenbrikettierung. - Braunkohle 32, S. 901-904, Halle a. d. S. 1933. - - Kennziffern für Siebanalysen und Zahlentafeln ähnlicher Art. - Glückauf 69, S. 533-537, Essen 1933. - Kennziffern für Siebanalysen. Hausmitteilungen d. Fa. L. Herrmann, Dresden, 1. Jg. H. 2, S. 31-46, Dresden 1938.

Dammer, Br.: Über Flottsande in der östlichen Mark Brandenburg. - Jb. Reichsstelle f. Bodenforsch. 61, S. 186-197, Berlin 1941

FAuler, W.: Der Löß und Lößlehm des Schwarzwaldrandes zwischen Achern und Offenburg. N. Jb. Min. etc. BB. 75, Abt. B. S. 191-230, Stuttgart 1936.

Grahmann, R.: Zum Vortrag von Herrn Dr. Breddin: Löß, Flugsand und Niederterrasse im Niederrheingebiet, ein Beitrag zur Frage der Entstehung des Löß. - Geol. Rdsch. 18, S. 298-299, Berlin 1927. - - Der Löß in Europa. - Mitt. Ges. Erdkde. Leipzig 51, S. 5-24, 3 Taf., Leipzig 1932.

Hohenstein, V.: Die Löß- und Schwarzerdeböden Rheinhessens. - Jb. u. Mitt. oberrhein. geol. Ver., N.F. 9, S. 74-97, Stuttgart 1920.

Scheidig, A.: Der Löß und seine geotechnischen Eigenschaften. - 233 S., Steinkopff, Dresden 1934.

Schnell, A.: Chemische Untersuchungen über den Iöß von Sprendlingen (Rheinhessen). - Diss., Gießen 1928.

SснӧNhals, E.: Gesetzmäßige Beziehungen zwischen Körnung uud Kalkgehalt des Lösses und die Erkennung von Verwitterungszonen mit Hilfe der typischen Streubereiche. - Geol. Jahrb. 66, S. 291-304, Hannover 1952. - - Gesetzmäßigkeiten im Feinaufbau von Talrandlössen mit Bemerkungen über die Fntstehung des Lösses. - Eiszeitalter und Gegenwart 3, S. 19-36, Ơhringen 1953.

Sмiтh, G. D.: Illinois Loess, Variations in its Properties and Distribution. - Univ. Illinois, Agricultural Experiment Station, Bull. 490, S. 139-184, mit kolorierter Karte, Urbana, Illinois 1942.

Woldstedt, P.: Das Eiszeitalter. Grundlinien einer Geologie des Quartärs. 1. Bd., 2. Aufl., 374 S., Stuttgart 1954.

ZINK, Fr.: Zur diluvialen Geschichte des Hochrheines und zur Altersstellung der paläolithischen Station "Murg“. - Mitt. Reichsstelle f. Bodenforsch., Zweigstelle Freiburg i. Br. H. 1, 51 S., Freiburg 1940.

Manuskr. eingeg. 28. 3. 1955.

Anschrift des Verf.: Privatdozent Dr. E. Schönhals, Bezirksgeologe beim Hessischen Landesamt für Bodenforschung, Wiesbaden, Mainzer Straße 25. 